\title{
Distributed quantum dot sensors for monitoring the integrity of protective aerospace coatings
}

\author{
A. Trinchi*, T.H. Muster, S. Hardin, D. Gomez, I. Cole, P. Corrigan, A. Bradbury \\ 1 CSIRO Materials Science \& Engineering \\ Gate 5, Normanby Road \\ Clayton, VIC 3168, Australia \\ $+61-3-95452777$ \\ adrian.trinchi@csiro.au \\ T.-L. Nguyen \\ School of Chemistry and Bio21 Institute, \\ The University of Melbourne, Parkville, Victoria, 3010, Australia. \\ M. Safai, G. Georgeson, D. Followell \\ Support \& Services Technology \\ Boeing Research \& Technology
}

\begin{abstract}
The development of a non-destructive healthmonitoring system for painted metal structures is being pursued. The system is based on sensing the fluorescence emission intensity of quantum dot particles that have been distributed throughout protective aerospace coatings. The quantum dots serve as distributed sensors that can provide localized information on the corrosive conditions that exist within the paint film. Consequently this provides an early indication of several key aspects, which include: the quality of film application; the in-service loss of corrosion inhibitor; and the mechanisms of failure.
\end{abstract}

In this paper we present the successful distribution of $\mathrm{CdSe} / \mathrm{ZnS}$ core-shell nanoparticles into a commercial chromate-inhibited aerospace primer and demonstrate a correlation between available levels of hexavalent chromium corrosion inhibitor and quantum dot photoluminescence during accelerated corrosion testing. The development of a non-destructive inspection device utilizing a microchannel gated intensifying camera is in progress. Finally, we discuss the limitations of available quantum dot materials and provide a vision for non-destructive corrosion inspection in metal structures.

\section{TABLE OF CONTENTS}

1. INTRODUCTION

2. METHODS AND EXPERIMENTAL .....................................2

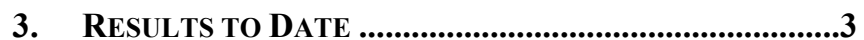

4. CONCLUSIONS ............................................................7

5. REFERENCES ...................................................... 7

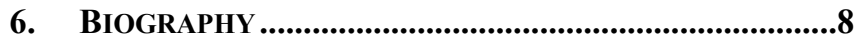

7. ACKNOWLEDGEMENTSERROR! BOOKMARK NOT DEFINED

\section{INTRODUCTION}

The costs associated with corrosion are significant, and mainly arise due to expensive maintenance routines [1]

. 978-1-4577-0557-1/12/\$26.00 C2012 IEEE
The ability to detect corrosion, or better still, the corrosive conditions before the onset of corrosion, has benefits for safety and the planning of maintenance [2][3]. Many aerospace components are fabricated from aluminium alloys and possess a protective coating system where a chromateinhibited primer is applied over the top of a synthetic oxide coating (conversion coating or anodized coating) [4][5] The later is a low-solubility oxide that promotes adhesion between the alloy and primer. The primer layer is typically an organic epoxy-polyamide matrix containing up to $40 \% \mathrm{w} / \mathrm{w}$ of sparingly soluble hexavalent chromium pigments (i.e. $\mathrm{SrCrO}_{4}$ ). Hexavalent chromium is an extremely effective corrosion inhibitor and prevents the pitting of aluminium alloys when supplied in concentrations exceeding $10^{-5} \mathrm{M}[6][7]$.

The corrosion on metal substrates frequently involves the formation of localised regions of high and low $\mathrm{pH}$, corresponding to cathodic and anodic corrosion processes, respectively. This is especially true on aerospace $\mathrm{Al}$ alloys such as AA2024-T3, where galvanic corrosion is set up as a result of the presence of $\mathrm{Cu}$-rich intermetallics which are cathodic relative to the matrix.

Obtaining information on localised corrosion states across a very large sample is inherently challenging, and hence the development of a distributed corrosion sensing system that is incorporated into the paint film will enable appropriate maintenance that may not only extend and/or identify the protective life-time of the protective aerospace coating, but may also dramatically reduce maintenance costs.

One such approach may involve the utilization of quantum dots (fluorescent semiconductor nanoparticles) to serve as corrosion sensors distributed throughout the protective aerospace coating. The implementation of such an approach comprises several scientific challenges, which include; the successful distribution of quantum dots into paint matrices, 
determining the relationships between the quantum dot photoluminescence and the corrosion or degradation of the coating, the development of a non-destructive interrogation and detection technique for the quantum dots in the paint, and the meeting of environmental challenges associated with materials selection.

This work is concerned with developing an understanding of the intricacies of using quantum dots to follow the degradation state of aerospace primers. The stability of quantum dots may be influenced by a number of factors, including: temperature, moisture, salts and other ions/chemicals, primer formulation, UV exposure. This study focuses on the behaviour of $\mathrm{CdSe} / \mathrm{ZnS}$ particles in harsh corrosive environments, and provides some insight on temperature, moisture, acidity/alkalinity and salinity response. As will be shown, photoluminescence from quantum dots is decreased under acidic conditions, and is possibly accelerated in the presence of hexavalent chromium. Thus the sensitivity of quantum dots to corrosion conditions provides the opportunity to develop early warning sensing for coating degradation and potential corrosion damage. Approaches to detecting photoluminescence have been considered and possibilities and opportunities are discussed within.

\section{METHODS AND EXPERIMENTAL}

\section{Quantum dot (QD) materials:}

The quantum dots used in this work were red-emitting $\mathrm{CdSe} / \mathrm{ZnS}$ core shell particles dispersed in either chloroform or water. The chloroform dispersible octadecylamine capped QDs were synthesized using previously reported methods [8][9]. Water dispersible QDs were prepared by encapsulating the octadecylamine capped QDs with amphiphilic poly(styrene-co-maleic anhydride) and ethanolamine (PSMA-EA). This surface functionalisation technique utilises ethanolamine as the malei anhydride ringopening agent to expose the hydrophilic carboxylic groups on the polymer [10] These red-emitting QDs possess a first exciton absorption peak maximum at $632 \mathrm{~nm}$ and an emission peak at $647 \mathrm{~nm}$ with FWHM $=25 \mathrm{~nm}$. The average particle diameter was $9.0+/-0.5 \mathrm{~nm}$, as established using Transmission Electron Microscopy.

Table 1. Composition of Sterling U-1201 aerospace resin.

\begin{tabular}{lc}
\hline Component & Concentration (\%weight) \\
\hline Methyl isobutyl ketone & $20-25$ \\
Strontium chromate $\left(\mathrm{SrCrO}_{4}\right)$ & 20 \\
Toluene & $15-20$ \\
Amorphous silica & 5 \\
Titania & $<5$ \\
Crystalline silica & $<0.2$ \\
Polymer component & Balance \\
\hline
\end{tabular}

$\mathrm{CdSe} / \mathrm{ZnS}$ quantum dots were dispersed into the resin component of a commercial aerospace primer (Sterling, U1201) together with the curing agent/catalyst (U-1202). The viscosity of the mixture was lowered, for the purpose of spin coating onto the aluminium panels, using U-1288 thinner. Details of the composition of the resin are given in

Table 1. A 1:1:1 mixture (by weight) of resin, catalyst and thinners was utilised for spin coating onto the aluminium panels.

\section{Coating Preparation:}

The Sterling primer coatings on polished AA2024-T3 panels were achieved using a spin-coater (Headway Research Inc. Photoresist Spinner, Model EC101). The surfaces of AA2024-T3 panels were cleaned prior to coating by lightly rubbing with a lint-free tissue soaked in ethanol. No surface activation or conversion coating was used in this work. Spin coating was achieved by placing 7 droplets of the quantum dot loaded primer solutions onto the AA2024-T3 then immediately starting the spinner and rotating at $2000 \mathrm{rpm}$ for $20 \mathrm{~s}$. Immediately after spinning, the samples were placed into a sealed storage container (to avoid dust collection) for further curing.

Table 2 lists the concentrations of $\mathrm{CdSe} / \mathrm{ZnS}$ core-shell quantum dot solutions that were prepared for spin coating. It also provides estimated amounts of quantum dots contained in the prepared samples. Prior to depositing the primers onto the aluminium panels, the $\mathrm{CdSe} / \mathrm{ZnS}$ in chloroform solutions were dispersed into the Primer Resin by shaking rapidly with a laboratory test tube mixer $\left(\mathrm{IKA}^{\circledR}\right.$ Labdancer vortex mixer) for three minutes prior to spin coating.

Table 2. Details of quantum dot coating solutions and final properties of spin coatings applied to polished AA2024-T3 specimens .

\begin{tabular}{cccc}
\hline $\begin{array}{c}\text { Sample } \\
\text { type }\end{array}$ & $\begin{array}{c}\text { Quantum dot } \\
\text { concentration in } \\
\text { resin }(\mathrm{mol} / \mathrm{l} \mathrm{CdSe})\end{array}$ & $\begin{array}{c}\text { Estimated } \\
\text { coating QD } \\
\text { mass }\left(\mathrm{g} / \mathrm{m}^{2}\right)\end{array}$ & $\begin{array}{c}\text { Estimated } \\
\text { density } \\
\left(\mathrm{QD} / \mathrm{m}^{2}\right)\end{array}$ \\
\hline P0 & 0 & 0 & 0 \\
P1 & $6.9 \times 10^{-7}$ & $6.9 \times 10^{-6}$ & $1.0 \times 10^{13}$ \\
P2 & $1.7 \times 10^{-6}$ & $1.7 \times 10^{-5}$ & $2.5 \times 10^{13}$ \\
P3 & $2.6 \times 10^{-6}$ & $2.6 \times 10^{-5}$ & $3.9 \times 10^{13}$ \\
\hline
\end{tabular}

Fluorescence spectra were recorded using either a Cary Eclipse Fluorescence Spectrophotometer (Varian) or a Raman Microscope (Renishaw). For liquid samples the fluorescence excitation and emission spectra were collected from an incidence angle of $90^{\circ}$. Both glass and quartz cuvettes were used. Reflectance measurements from solid surfaces were achieved using an incidence angle of $45^{\circ}$ relative to the sample surface. Aqueous-based samples were excited at wavelength of $400 \mathrm{~nm}$, whilst a $500 \mathrm{~nm}$ excitation wavelength was used for the reflectance measurements. 
Raman spectra and maps of the quantum dot loaded primer coatings were collected using a Renishaw inVia confocal microscope system. Specimens were illuminated through a $50 \times$ objective lens (numerical aperture of approximately 0.75 ) with $514 \mathrm{~nm}$ excitation coming from an argon ion laser source. The spot size was approximately $1.3 \mathrm{~mm}$. Spectra were collected over the spectral range of $550-1,250 \mathrm{~cm}^{-1}$.

\section{Results to DATE}

Incorporation of quantum dots into aerospace primer:

Figure 1 shows the fluorescence emission spectra of AA2024-T3 samples coated with the Sterling primer without quantum dots ('P0') and with loadings corresponding to 'P1', 'P2' and 'P3' as described in Table 2. All samples exhibited good reproducibility in terms of their fluorescence spectra. Whilst peak intensities attributed to the commercial primer, occurring at $575 \mathrm{~nm}$ and $675 \mathrm{~nm}$, varied slightly, the peak at $647 \mathrm{~nm}$, which is the characteristic emission peak due to the incorporation of $\mathrm{CdSe} / \mathrm{ZnS}$ quantum dots, showed a systematic increase in fluorescence intensity with loading.

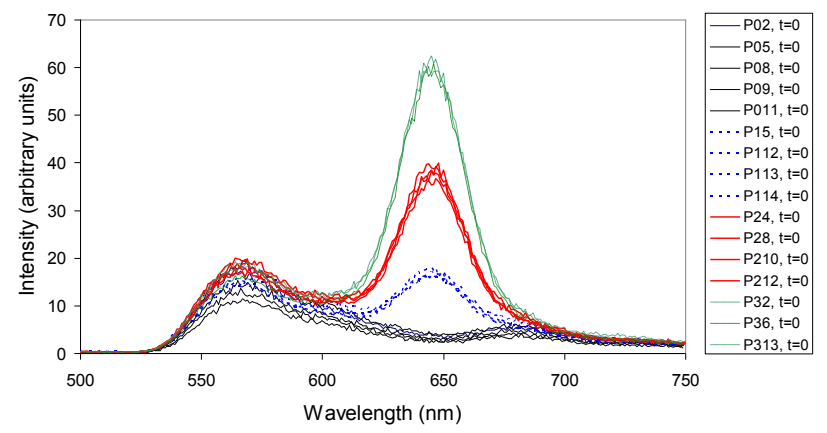

Figure 1 - Fluorescence spectra of replicates of primed AA2024-T3 panels. Samples P0x contain no quantum dots. Samples $P 1 x, P 2 x$ and $P 3 x$ contain incremental quantum dot loadings according to Table 2. Excitation at $500 \mathrm{~nm}$.

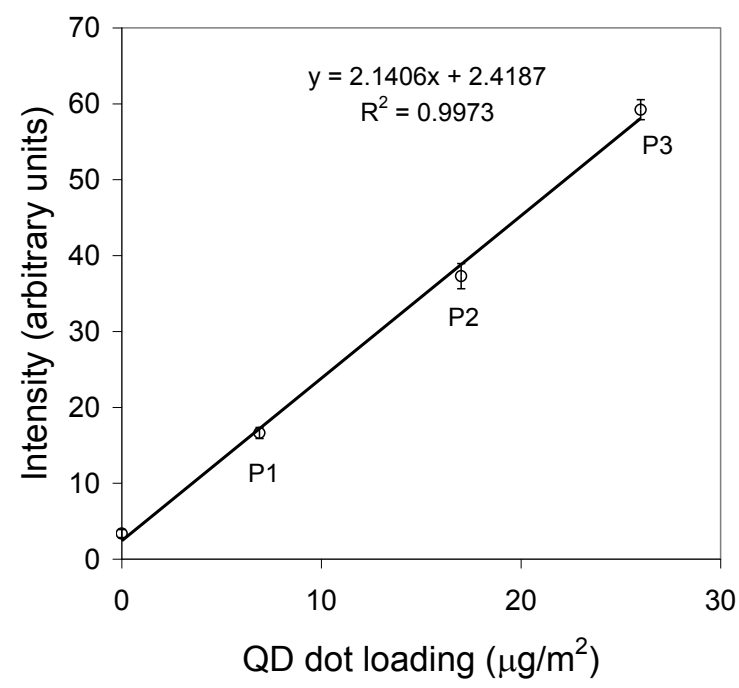

Figure 2 - Fluorescence emission at $647 \mathrm{~nm}$ with incremental loading of quantum dots into aerospace primer.

Figure 2 shows that the fluorescence emission intensity (in arbitrary units) plotted against the quantum dot loading (per $\mathrm{cm}^{2}$ ) exhibits a linear relationship. This suggests that quantum dots are dispersed evenly into the primer systems and that there are no losses in fluorescence emission with increased quantum dot loading. Since quantum dot incorporation into the PMMA and primer-based coatings appeared to be reproducible, this established a sound baseline for further degradation studies.

\section{Accelerated corrosion testing:}

The average fluorescence emission spectra of the Sterling primer containing $\mathrm{CdSe} / \mathrm{ZnS}$ quantum dots, after having spent $0,1,10$ and 25 days in the accelerated testing chamber are seen below in Figure 3. For the P0 samples (those containing no quantum dots, Figure $3 a$ ) the decrease in the paint peak intensities occurring at $568 \mathrm{~nm}$ and $682 \mathrm{~nm}$ was only gradual with time, due to coating degradation over time. For samples P1 through to P3 Figure 3b-d) after one day of accelerated testing the quantum dot peak at $647 \mathrm{~nm}$ remains quite prominent throughout the spectrum, and decreases significantly by day 10 . Interestingly, the peak at $647 \mathrm{~nm}$ seems to have all but disappeared in each of the samples by day 25 . 

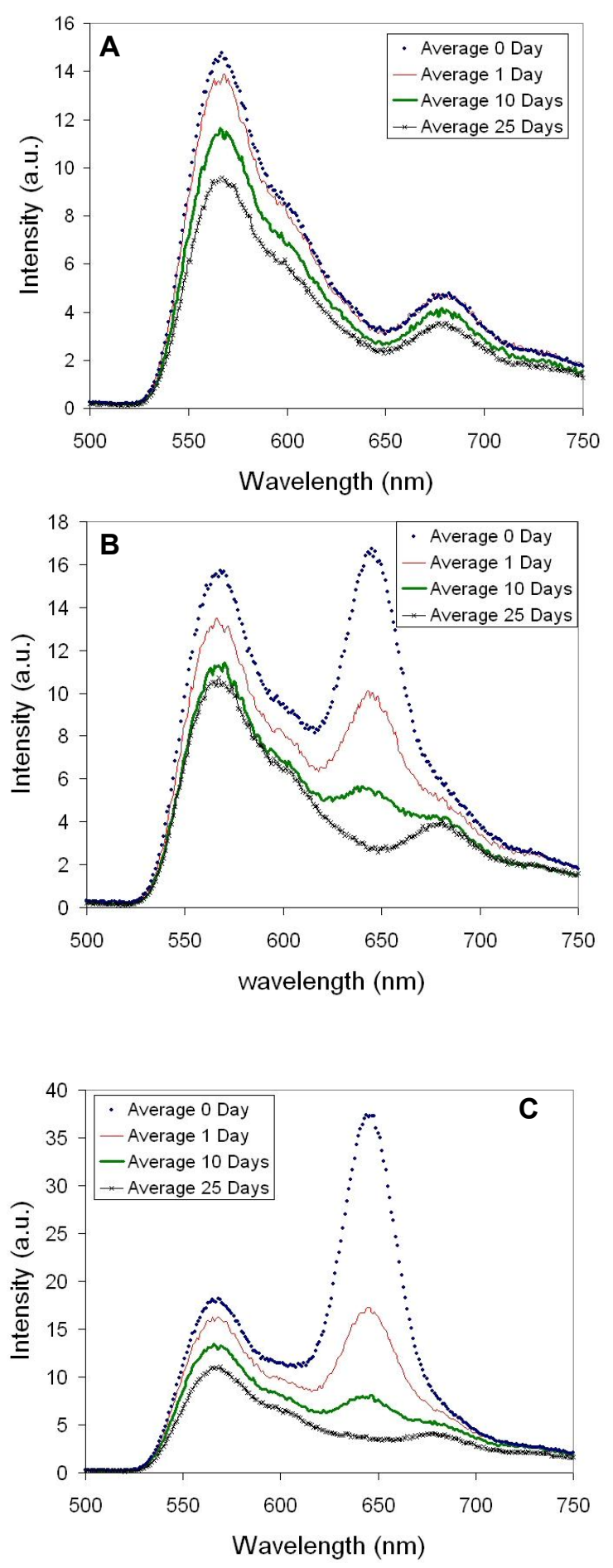

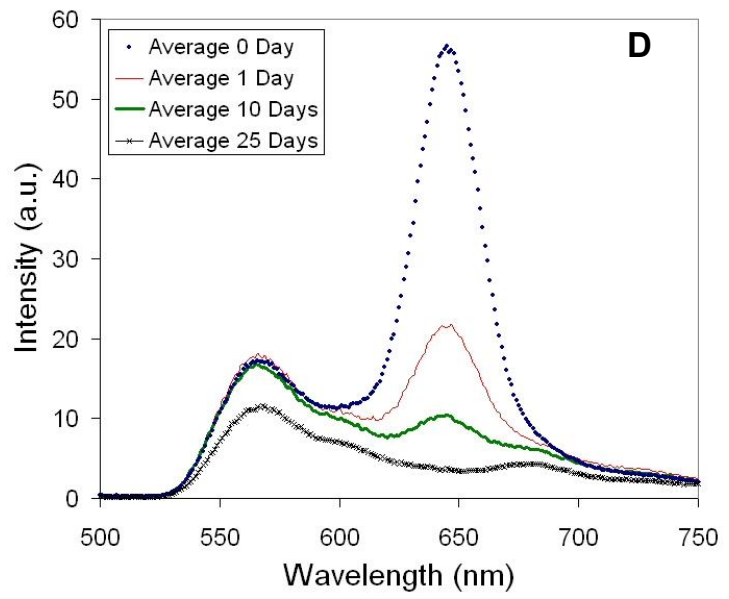

Figure 3 - Average fluorescence emission spectra of the Primed QD samples after different exposure times in the Ascott Chamber. (A) $=$ P0, (B) $=$ P1, (C) $=$ P2, (D) $=$ P3.

A plot of the fluorescence emission at $647 \mathrm{~nm}$ after different exposure times for the Primed QD samples in seen in Figure 4 , and suggest for each of the samples there is an exponential decrease in the peak intensity with exposure time that is proportional to the concentration of quantum dots in the sample. All the quantum dots in primer samples showed near complete loss of fluorescence signal after 25 days exposure.

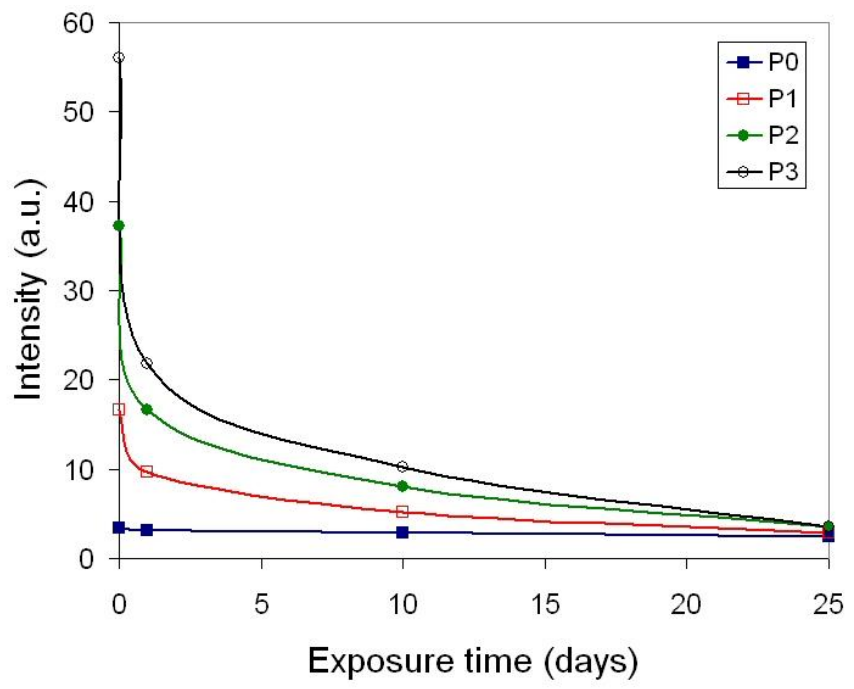

Figure 4 - Fluorescence emission at $647 \mathrm{~nm}$ after different exposure times in the Ascott Chamber for the Primed QD samples.

Raman spectroscopy was performed on representative areas of P3 samples both prior to, and following accelerated corrosion testing. Both Figure 5 and Figure 6 and demonstrate that Raman analysis is sensitive to both chromate concentrations and quantum dot emission. This enabled a direct comparison to be made between the effective protection provided by the aerospace primer, as 
detailed by the remaining corrosion inhibitor in the primer, and the fluorescence from $\mathrm{CdSe} / \mathrm{ZnS}$ quantum dots. As Figure 7 shows, this relationship as a first approximate appears to be linear, suggesting for the coating preparations used in this study, that quantum dot luminescence could be directly utilized to determine the effective remaining life of primer. The horizontal dashed line in Figure 7 suggests that where chromate levels are depleted below a certain critical concentration the inhibitor will be no longer able to prevent pitting corrosion of the underlying metal, hence sensing of quantum dot photoluminescence may provide an early indication of potential failure.

The intense photoemission from quantum dots implies they are extremely attractive for highly sensitive monitoring of changes in their chemical environment. As a demonstration of this one may compare the spectra in Figure 5 and in Figure 6.and, the Chromate peak occurring at $867 \mathrm{~cm}^{-1}$ has an intensity of 2000 when excited using $100 \%$ of $10 \mathrm{~mW}$ laser, whereas quantum dots have an emission intensity of 60,000 when excited by $1 \%$ of $10 \mathrm{~mW}$.

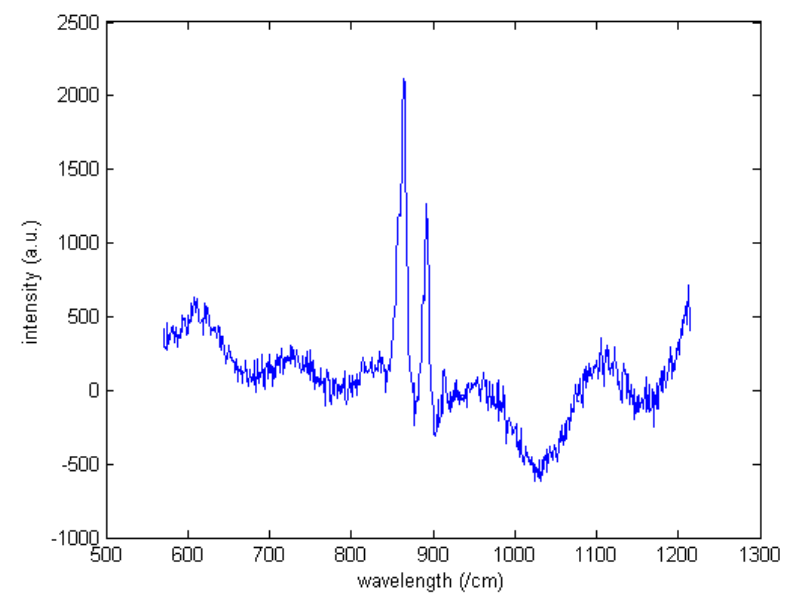

Figure 5 - Raw Raman spectra of chromate.

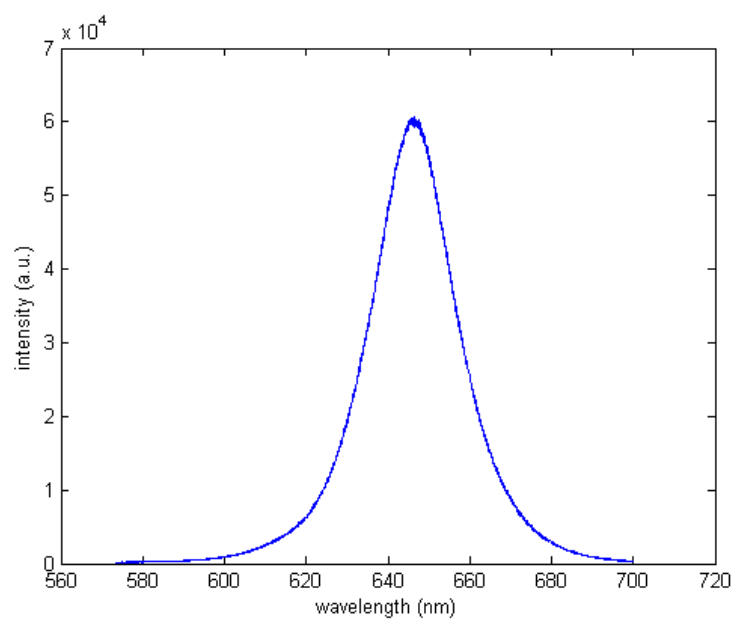

Figure 6 - Fluorescence spectrum of the CdSe/ZnS quantum dots obtained with the Raman.

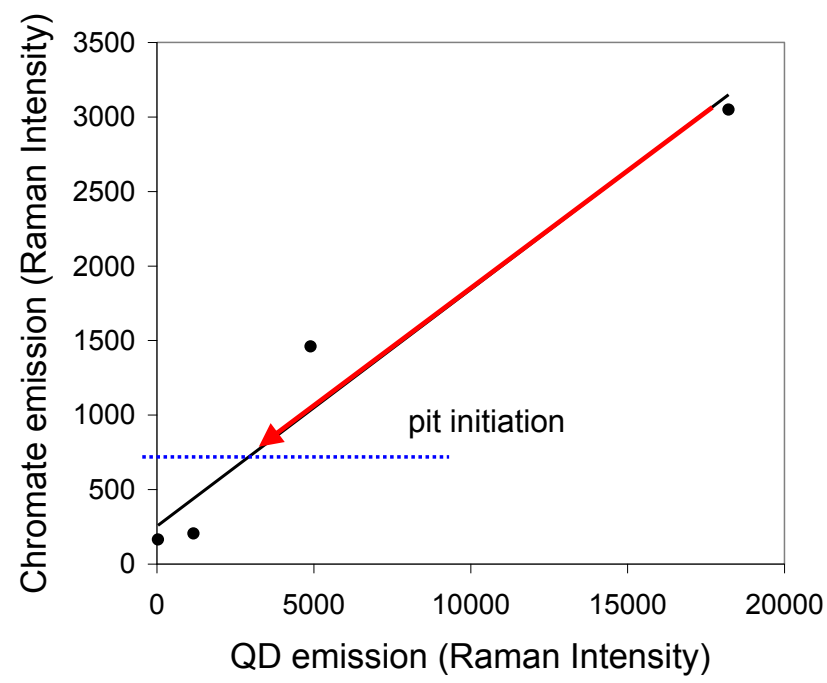

Figure 7 - Chromate peak intensity at $894 \mathrm{~cm}-1$ plotted against CdSe/ZnS quantum dot emission for P3 sample before and after accelerated corrosion testing at 1, 10 and 25 days (solid dots).

Aqueous stability of CdSe/ZnS quantum dots:

The rapid deterioration of photoluminescence intensity during accelerated corrosion testing (Figure 4) suggests that the $\mathrm{CdSe} / \mathrm{ZnS}$ quantum dots used in this work are susceptible to degradation upon exposure to aqueous environments. Whilst the aqueous chemistry within an aerospace primer is not well known, quantum dots are likely to be exposed to significant concentrations of $\mathrm{Cr}^{6+}$, and where corrosion occurs on metal substrates, localized regions of anodic and cathodic reactions lead to the formation of acidic and alkaline zones. Here the rapid loss of both chromate and photoluminescence may be a consequence of using a low and variable primer thickness (6 $\pm 4 \mathrm{~m}$ ) that supported high rates of water ingress and mass transport of soluble ions out of the coating. An alternative theory is that hexavalent chromium may be responsible for oxidising CdSe upon hydration of the coating. For instance:

$$
2 \mathrm{Cr}^{6+}+\mathrm{CdSe} \rightarrow 2 \mathrm{Cr}^{3+}+\mathrm{Cd}^{2+}+\mathrm{Se}^{4+} \quad \ldots 1
$$

By virtue of the $\mathrm{ZnS}$ shell present in the quantum dots used in the current work, it would be expected that the quantum dots would undergo degradation in sufficiently acidic environments, such as would arise during active corrosion. The dissolution of sulphides in acid media is a well documented phenomenon [11]; removal of the $\mathrm{ZnS}$ shell in the quantum dots would lead to loss of specific fluorescence of the nanoparticles. As a model for such processes, the fluorescence stability of the CdSe/ZnS QD's was tested in buffers at $\mathrm{pH} 2,4,7$ and 10. Fluorescence intensity was 
monitored as a function of time and data are presented in Figure 8 .

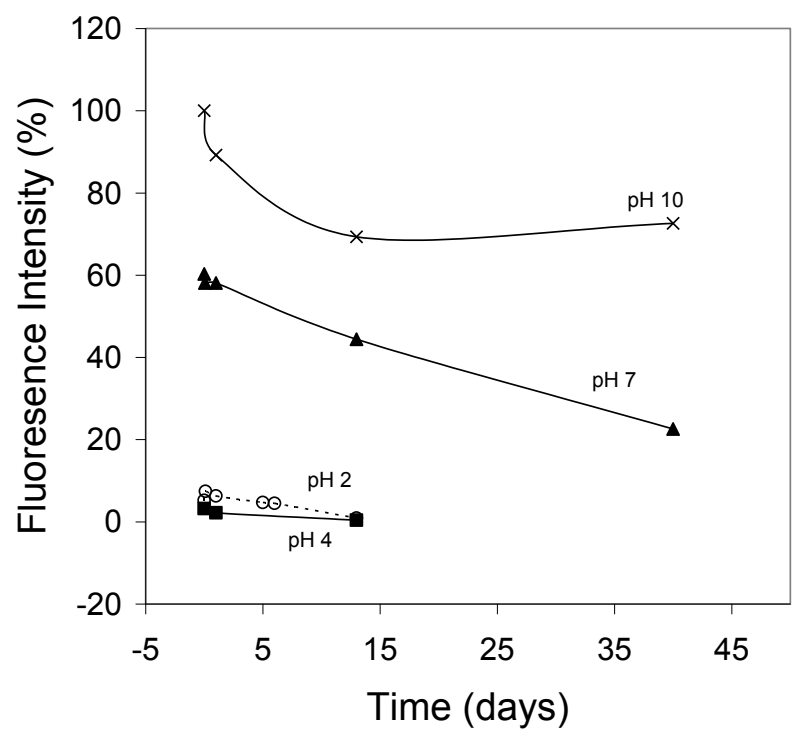

Figure 8 - Fluorescence intensity of $1 \% \mathrm{v} / \mathrm{v}$ CdSe QD at $646 \mathrm{~nm}$ as a function of $\mathrm{pH}$ and time.

The samples investigated at $\mathrm{pH} 2$ and $\mathrm{pH} 4$ underwent immediate coagulation and degradation to a brownish precipitate immediately after preparation. The $\mathrm{pH} 7$ solution was initially clear, but steadily developed a precipitate over time. When subjected to acidic conditions, $\mathrm{pH} 2$, the quantum dots underwent immediate decomposition with almost total loss of fluorescence. The decomposition at $\mathrm{pH} 4$ was also rapid, with the fluorescence emission intensity decreasing by $90 \%$ immediately, but a small amount of residual fluorescence remained for up to 2 weeks.

On the other hand, when subjected to more alkaline conditions, $\mathrm{pH} 10$, the solution showed the best stability, displaying only a small amount of precipitate even after 40 days. Samples at $\mathrm{pH} 7$ and $\mathrm{pH} 10$ retained significant fluorescence for at least 40 days. The $\mathrm{pH} 7$ sample showed an immediate decrease in fluorescence intensity of about $40 \%$ of (relative to the $\mathrm{pH} 10$ sample) and thereafter declined almost linearly with time to about $20 \%$ intensity after 40 days. The $\mathrm{pH} 10$ sample declined to about $70 \%$ of initial intensity by 2 weeks, then stabilised thereafter. Over time, both the $\mathrm{pH} 7$ and $\mathrm{pH} 10$ samples displayed increasing peak broadening, in particular those held at $\mathrm{pH} 7$. However, all maxima remained centred near $646 \mathrm{~nm}$.

The study of the fluorescence of water-soluble PSMA capped $\mathrm{CdSe} / \mathrm{ZnS}$ demonstrated their vulnerability to acidic conditions. This is not unexpected as the PSMA/EA groups on the QD surface partially relies on the carboxyl groups being ionised to electrostatically stabilise the QDs. At low $\mathrm{pH}$ values, when the carboxyl groups are protonated, the polymer no longer confers colloidal stability of the QDs. At high $\mathrm{pH}$ values however, the peak broadening and coagulation of the QD solutions could be due to electrostatic coupling of free amines to the carboxyl groups. It has been shown that when PEG groups are used to ring-open the maleic anhrydride, the PSMA/PEG coated QDs are stable over a wider range of $\mathrm{pH}$ [10]. This type of surface functionalisation on the QDs would be more suitable for the purpose of this study.

Non-destructive detection of quantum dot photoluminescence:

In order to have a field deployed system for the detection of quantum dots in the paint films a quantum dot corrosion detection system that will have a gated intensifying camera sensor, and a gated laser excitation system is under development (Figure 9). The system will be integrated in a vacuum hood, in order to be shielded from unwanted radiation which can get into detection system, and will also reduce the effect of moisture and pollution. Additionally this approach will eliminate the need for a narrow band pass optical filter which will tend to reduce the signal intensity. Most quantum dot fluoroscopy to-date has been demonstrated in controlled scientific laboratories. Different types of detection methods are being used, such as laser excitation pump and probe with phase lock detection and gated imaging. All these techniques have shown the ability to detect the shift in the wavelength of the quantum dots.

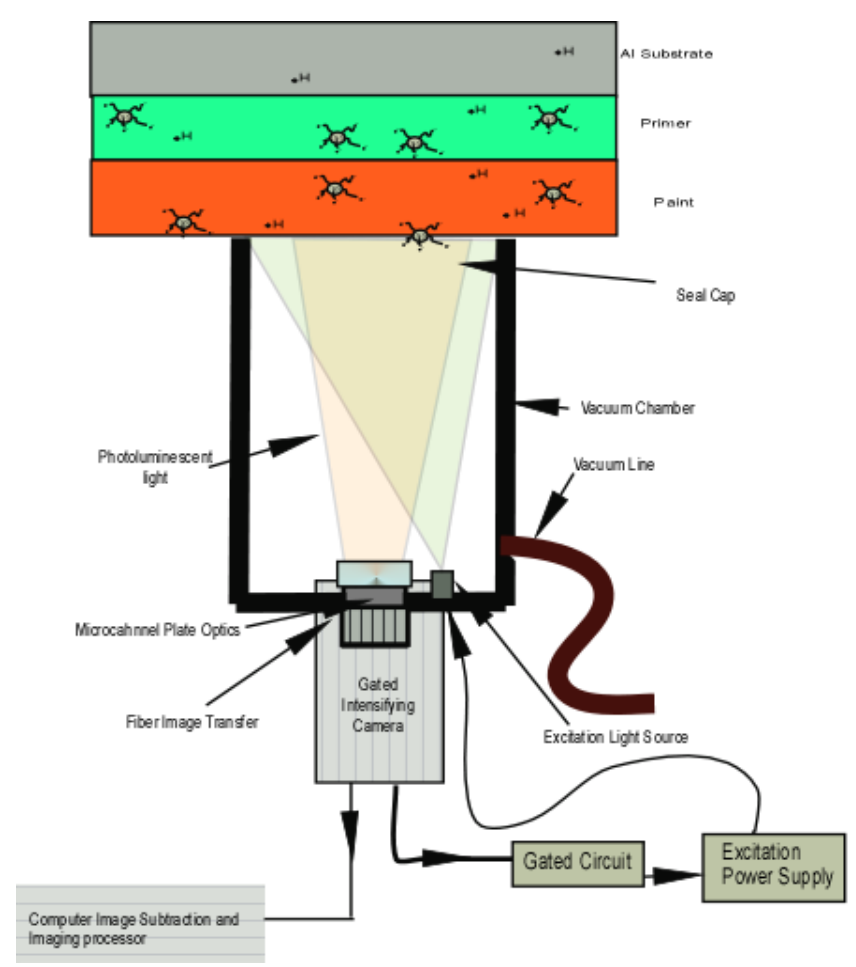

Figure 9 - Demonstration Quantum Dot Fluoroscopic Corrosion Detection System..

A low noise intensifying camera such as the one manufactured by Hamamatsu Inc. will be used for the laboratory demonstration. This intensifying camera contains 
a gated image intensifier, fiber-bundle based imaging transfer technology, and a 2-D imaging sensor. The detection system will also encompass a photo excitation source, such as laser diode or LED in order to excite the quantum dots into a higher energy state. The selection of an intensifying camera will depend upon the wavelength of the specific quantum dot photoluminescence characteristics. The excitation source and the image intensifier will be gated such that the illumination of excitation source is turned off during the image intensifier reading. This technique can be modulated at very high speed if needed (10 nanosecond gating) in order to insure the detection of the short excitation life of quantum dot photoluminescence. Also, this technique eliminates the need for signal-reducing optical filtering.

Two types of systems are being investigated. A quantum dot based corrosion monitoring system will require the development of a wireless or fibre optic MEMS sensor network for SHM applications. A portable inspection system for in-service NDE applications could also be developed, which would not require MEMS development. The SHM system is the best long-term corrosion monitoring system.

\section{Future challenges:}

CdSe is a useful material for research purposes due to its emission in the visible spectrum, however, one of the major challenges in implementing quantum dot technologies is minimizing the environmental impacts associated with cadmium release. Despite the small quantities required in developing sensing materials cadmium selenide, along with several other semiconducting quantum dot materials (group II-VI, III-V and IV-VI) may be limited due to toxicity issues [12].

In the visible spectrum the there are several candidate materials that may be worth considering as alternatives to CdSe. Phosphides (such as aluminium phosphide), silicon, nanodiamond, and selected oxides and sulphides are all potential candidates. With respect to oxides, cobalt for instance, is able to emit at wavelengths $<900 \mathrm{~nm}$. Copper oxide is also a possibility due to its emission $<1000 \mathrm{~nm}$, however, copper is known to be detrimental to the corrosion performance of particular metals (i.e. aluminium alloys). Silicides such as $\mathrm{Mg}_{2} \mathrm{Si}$ may offer a vehicle to work in the near infrared region. In some cases it may be beneficial to work outside the visible spectrum as paint films can possess absorbance windows that would allow maximum penetration of incident and emitted light.

\section{Conclusions}

An approach for monitoring the degradation of aerospace primers that uses non-destructive interrogation of distributed quantum dots has been presented. It was shown that by monitoring the fluorescence intensity of the distributed quantum dots it is possible to track the degradation of primer coatings, in particular the remaining inhibitor concentration. Moreover, Raman microscopy investigation demonstrated that a linear correlation exists between the quantum dot photoluminescence and the hexavalent $\mathrm{Cr}$ concentration during accelerated corrosion testing using.

Progress has been made in understanding the dissolution properties of the quantum dots, which has strong relevance on their ability to be distributed into aerospace primer systems. In acidic conditions the CdSe quantum dots were shown to undergo rapid degradation, with the loss of fluorescence being attributed to the dissolution of its $\mathrm{ZnS}$ shell. Experimental design may remove, or lessen, many of these effects. Nevertheless, it appears that CdSe quantum dots are sensitive to acidic conditions and show considerable promise as $\mathrm{pH}$ probes to detect such conditions, especially anodic corrosion processes on aerospace $\mathrm{Al}$ alloys.

Implementation of quantum dot sensing technologies for paint monitoring will benefit from further developments in miniaturization of inspection devices, new quantum dot materials, and from greater control over quantum dot surface interactions. Our investigation thus far has shown that such an approach is feasible. The development of a nondestructive interrogation technique is in progress

\section{REFERENCES}

[1] N. Thompson, M. Yunovich, D. Dunmire, In: Proceedings of the 1st World Congress on Corrosion in the Military, Sorrento, Italy, June (2005).

[2] G. Bartelds, J.H. Heida, J. McFeat and C.Boller, In: Health Monitoring of Aerospace Structures: Smart Sensor Technologies and Signal Processing (eds W.J. Staszewski, C. Boller, G.R. Tomlinson), New York, John Wiley and Sons, 2004. pp 1-28.

[3] A. Trego, D. Price, M. Hedley, P. Corrigan, I. Cole, T. Muster, "Development of a system for corrosion diagnostics and prognostics", Corrosion Reviews, 25(1-2), 161-177, 2007

[4] A.E. Hughes, R.J. Taylor, B.R.W. Hinton, "Chromate conversion coatings on $2024 \mathrm{Al}$ alloy". Surface and Interface Analysis, 25, 223-234, 1997.

[5] M.W. Kendig, A.J. Davenport, H.S. Isaacs , "The mechanism of corrosion inhibition by chromate conversion coatings from $\mathrm{x}$-ray absorption near edge spectroscopy (Xanes)", Corrosion Science, 34, 4149p, 1993

[6] A.E. Hughes, I.S. Cole, T.H. Muster, R.J. Varley, "Designing green, self-healing coatings for metal protection", NPG Asia Mater. 2, 2010

[7] T.H. Muster, A.E. Hughes, S.A. Furman, T.G. Harvey, N. Sherman, S. Hardin, P. Corrigan, D. Lau, F.H. Scholes P.A. White, M. Glenn, J. Mardel, S.J. Garcia, J.M.C. Mol JMC, “A rapid screening multielectrode method for the evaluation of corrosion 
inhibitors", Electrochimica Acta, 54, 3402-3411, 2009

[8] B.O.Dabbousi, J. Rodriguez-Viejo, F.V.Mikulec, J.R.Heine, H.Mattoussi, R.Ober, K.F.Jensen and M.G.Bawendi, "(CdSe)ZnS core-shell quantum dots: synthesis and characterization of a size series of highly luminescent nanocrystallites", J.Phys.Chem. B, , 101, 9463-9475, 1997

[9] J. van Embden and P. Mulvaney, "Nucleation and growth of CdSe nanocrystals in a binary ligand system", Langmuir, 21, 10226-10233, 2005

[10] E.E. Lees, T.-L. Nguyen, A. H.A. Clayton and P. Mulvaney, "The preparation of colloidally stable, water-soluble, biocompatible semiconductor nanocrystals with a small hydrodynamic diameter", ACS Nano, 3, 1121-1128, 2009

[11]F.A. Cotton, G. Wilkinson, C.A. Murillo, M. Bochmann, "Advanced Inorganic Chemistry", New York, John Wiley \& Sons, 1999.

[12] A.M. Derfus, W.C.W. Chan, S.N. Bhatia, "Probing the cytotoxicity of semiconductor quantum dots", Nano Letters, 4, 11-18, 2004.

\section{BIOGRAPHY}

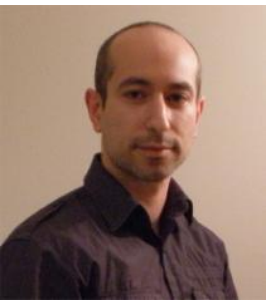

Dr Adrian Trinchi is a research scientist at CSIRO and works in the area of high throughput and combinatorial electrochemistry. Currently he is investigating new materials for corrosion and chemical sensing applications.

Dr Tim Muster is Team Leader of Functional interfaces and Senior Research Scientist. His work is focused on localized corrosion processes and the development of sensing technologies for corrosive environments.
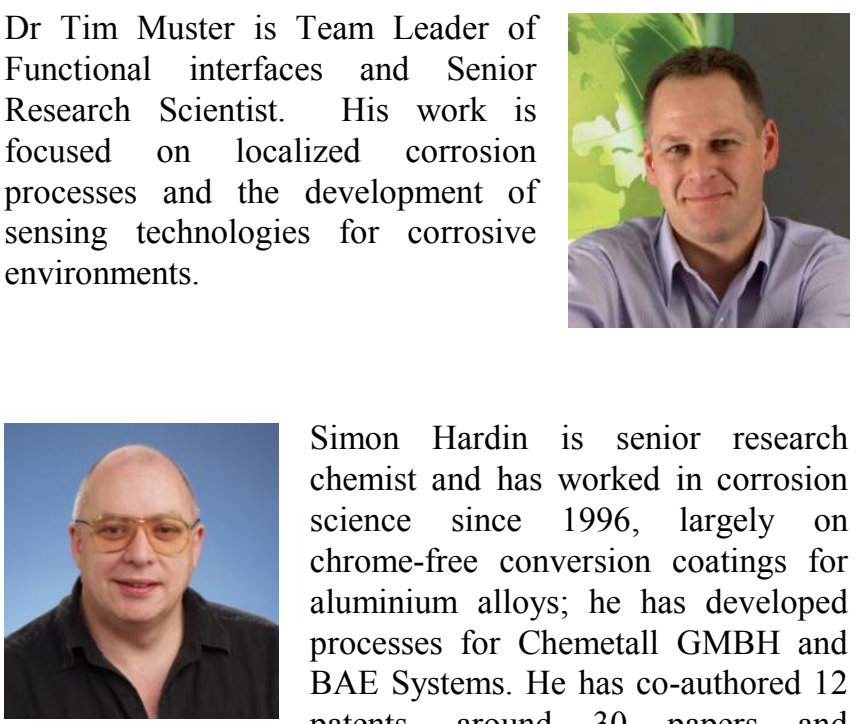

Simon Hardin is senior research chemist and has worked in corrosion science since 1996, largely on chrome-free conversion coatings for aluminium alloys; he has developed processes for Chemetall GMBH and BAE Systems. He has co-authored 12 patents, around 30 papers and numerous conference proceedings.
Dr Daniel Gomez is a Post-Doctoral research fellow at the CSIRO. He is currently working on incorporating quantum dots on plasmonic devices and developing an understanding of the optical properties of plamonic structures.
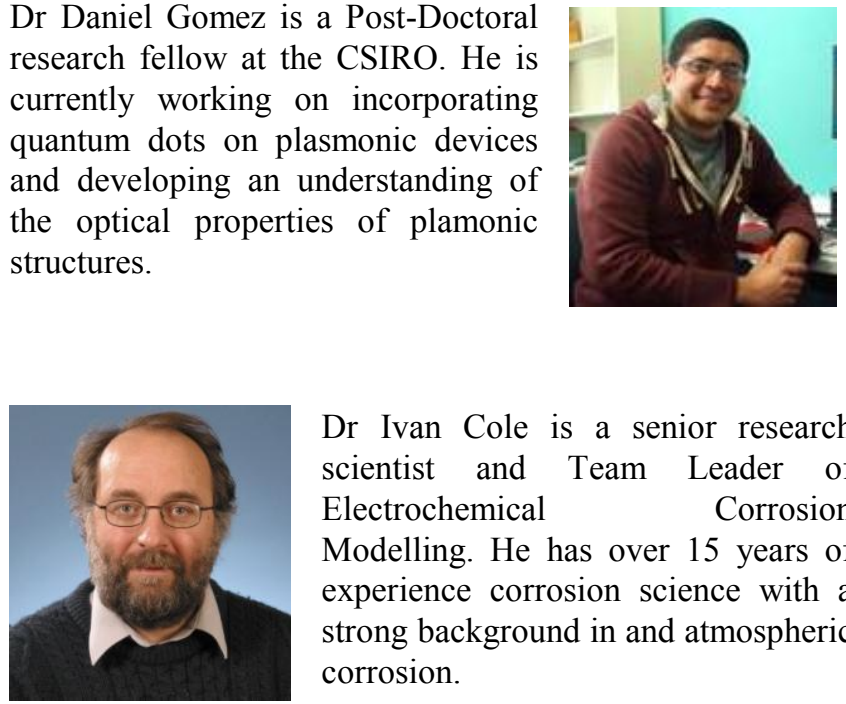

Dr Ivan Cole is a senior research scientist and Team Leader of Electrochemical Corrosion Modelling. He has over 15 years of experience corrosion science with a strong background in and atmospheric corrosion.

Penny Corrigan is a Project Leader and Research Scientist at CSIRO and is currently leading projects in the area of Intelligent Vehicle Health Monitoring (IVHM).
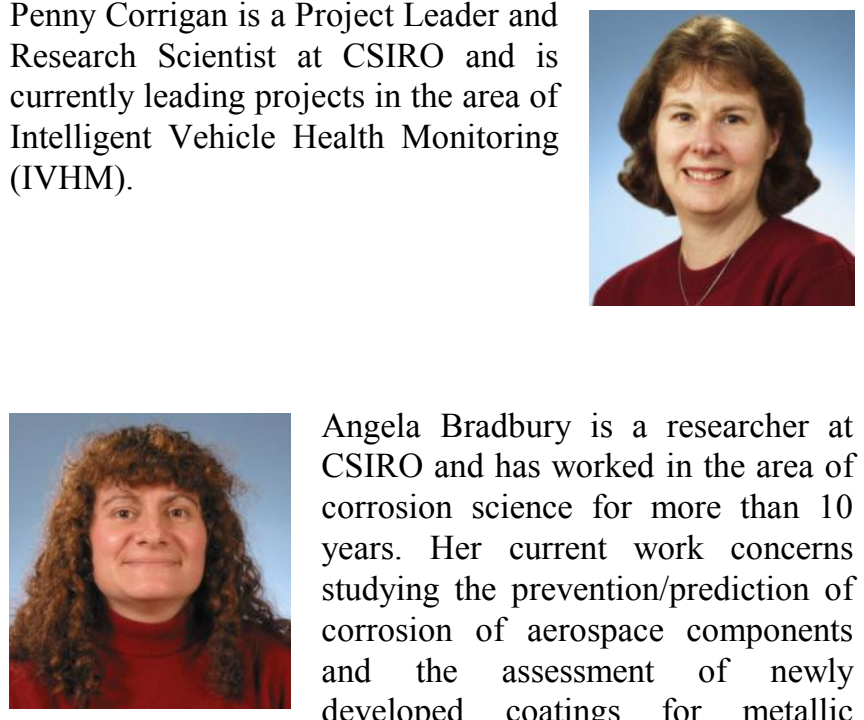

Angela Bradbury is a researcher at CSIRO and has worked in the area of corrosion science for more than 10 years. Her current work concerns studying the prevention/prediction of corrosion of aerospace components and the assessment of newly developed coatings for metallic components to prevent corrosion.

Dr Tich-Lam Nguyen is currently a research fellow in the Nanoscience Laboratory at the University of Melbourne. Her work mainly involves tailoring the structure of nanomaterials with interesting optoelectronic properties including the synthesis of metal colloids and quantum dots, and their surface functionalisation.
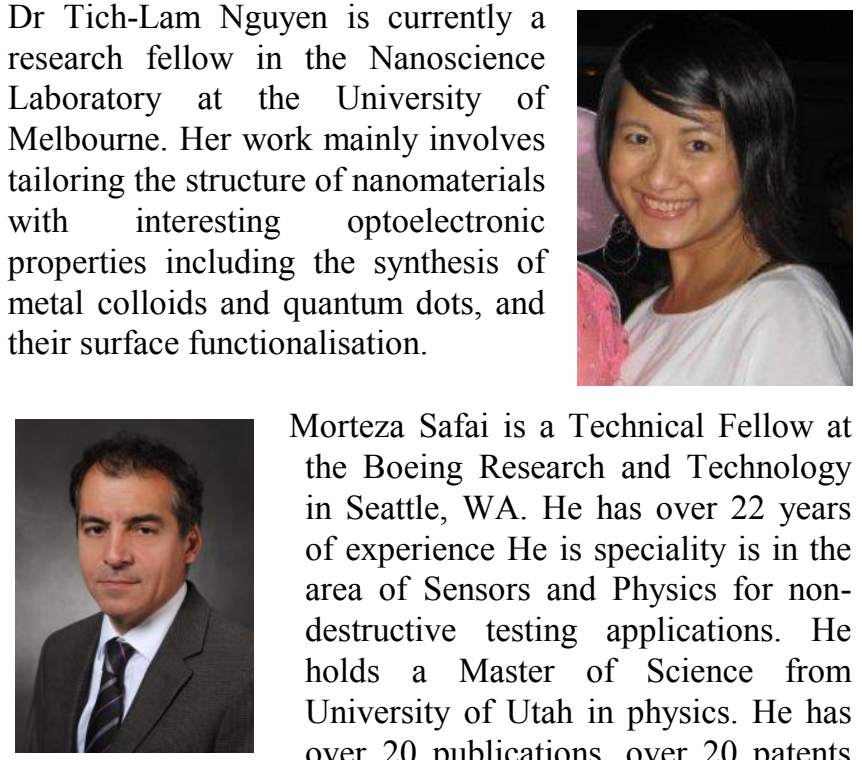

Morteza Safai is a Technical Fellow at the Boeing Research and Technology in Seattle, WA. He has over 22 years of experience He is speciality is in the area of Sensors and Physics for nondestructive testing applications. He holds a Master of Science from University of Utah in physics. He has over 20 publications, over 20 patents and over 30 patent pending. 


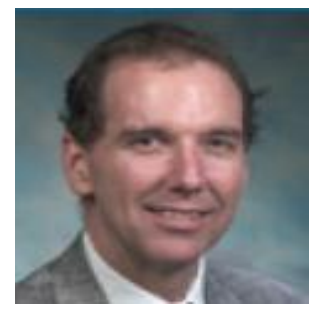

David Followell is a Technical Fellow within the Boeing Research \& Technology organization. He has been with The Boeing Company for 27 years working in technology areas which include vehicle health management, electronic packaging and reliability engineering. He holds a Bachelors of Science in Electrical Engineering.. He has published over 20 papers.

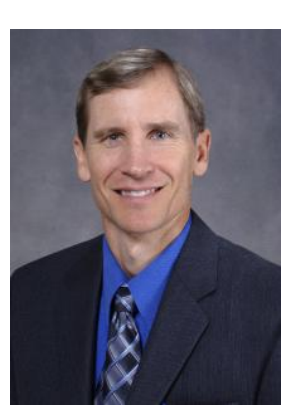

Dr. Gary Georgeson is a Technical Fellow for Boeing Research \& Technology in Non-Destructive Evaluation. $\mathrm{He}$ is recognized for developing innovative NDE methods for evaluating composite and metal aerospace structures. He has published or presented over 120 technical articles, and is a co-inventor on 75 patents, with over 50 patents pending. 